\title{
Do be so immature: dedifferentiated $\beta$ cells help mice evade Type 1 diabetes
}

Lee, H. et al. Cell Metabol 31, 822-836.e5 https://doi.org/10.1016/j.cmet.2020.03.002 (2020).

Not long after they were weaned, a cohort of Feyza Engin's mice became mildly hyperglycemic. That was unusual for animals so young, but the lab had just eliminated a component of their immune system with a suspected role in the development of Type 1 Diabetes. Perhaps this was a sign that the disease was just progressing faster in the absence of the gene they had knocked out, recalls Engin, a researcher at the University of Wisconsin Madison. But that first unusual observation was not to be the last.

Rather than continuing on a diabetic decline, their blood sugar soon returned to normal. At 16 weeks-when T cells would normally invade the pancreas of non-obese diabetic (NOD) mice and destroy their insulin-producing $\beta$ cells-Engin's animals remained healthy. The lab waited. And waited. And waited some more. " 50 weeks we waited," says Engin, monitoring the mice. "They were living happily and without any signs of diabetes."

Engin had brought the strain with her to Wisconsin from Harvard, where she had spent several years of her postdoc backcrossing generation after generation of mice to produce tissue-specific, drug-inducible diabetes models on a pure NOD background. Such models allows researchers to knockout different genes in a particular place and at a particular time. Engin is using the animals to study the role of genes that respond to stress in the development of Type I diabetes.

Type 1 diabetes is the result of an autoreactive immune system destroying insulin-producing $\beta$ cells in the pancreas. People can have a genetic predisposition to developing Type I diabetes, though there are environmental triggers that can influence its onset and progression, such as viral infections, toxins, and chronic inflammation. These can cause endoplasmic reticulum stress, which in turn prompts what's known as the unfolded protein response (UPR). There are several different genes involved in the UPR, which initially attempts to mitigate stress-induced damage to cells. With prolonged stress, however, the UPR changes course and will instead initiate cell death.

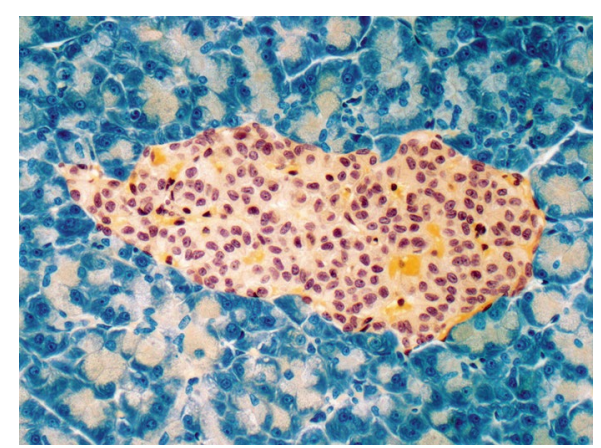

Sneaking by? In a mouse model of diabetes, dedifferentiated $\beta$ cells escape destruction by the immune system. Credit: STEVE GSCHMEISSNER/ Science Photo Library/ Getty

Engin has been studying how the $\beta$ cells themselves might contribute to their own demise. In prior work, she observed signs of endoplasmic reticulum stress in $\beta$ cells in two mouse models of diabetes as well as human patient samples; in the mice, mitigating that stress with a chemical chaperon called TUDCA (now in Phase II clinical trials for new-onset Type I diabetes) prevented disease onset. She is now using the inducible model to start digging into the molecular mechanisms.

In her latest paper, published in Cell Metabolism, Engin and her lab knocked out expression of inositol-requiring protein-1 alpha (IRE1 $\alpha)$, a gene involved in mediating inflammation and apoptosis or stress adaptation in the UPR, in the $\beta$ cells of pups just after birth. Eliminating IRE $1 \alpha$ - so early enabled the team to observe what was occurring in the mice before the immune system infiltrated the animals' $\beta$-cell containing pancreatic islets. After the lab spent that initial year waiting for diabetes that ultimately never developed, they set to work studying additional cohorts more closely.

During the animals' transient hyperglycemic phase, islet composition and morphology in the pancreas was considerably altered. Molecular analysis and single-cell sequencing of the IRE1 $\alpha$-lacking $\beta$ cells revealed that they were actually de-differentiating back to progenitor form-an adaptive means to minimize stress-induced damage that left behind immature $\beta$ cells unable to produce the insulin necessary to control the animals' blood sugar. As time went on however, those cells re-differentiated into their normal-functioning forms.

So why then did the T cells never come to recognize the re-differentiated cells? "When they came back, they should have been a target," says Engin. But in those de-differentiated $\beta$ cells, immune inhibitory marker genes were upregulated while those for auto-antigen expression were diminished. Lacking cues, the immune system was not being summoned to the pancreas.

Nor would the T cells have functioned normally, had they infiltrated the pancreas in the usual numbers. When the team transferred $\mathrm{T}$ cells from the knock-out animals to controls, the diabetes expected never developed. "The T cells' diabetogenic function was impaired," says Engin.

There are many questions still to answer. The lab is still working out the explanation for their non-diabetogenic $\mathrm{T}$ cell observation, along with developing a better understanding of the mechanisms involved in the $\beta$ cells' de- and re-differentiation and induction of immune tolerance. Engin hopes that such information might yield translational insights into Type 1 diabetes in humans, a condition diagnosed in tens of thousands of children and young adults each year that can be managed but not yet prevented or cured.

Should the same mechanisms found in mouse hold in humans, $\beta$ cell differentiation could be a critical therapeutic window for those at risk for Type 1 diabetes, Engin says. Follow up studies studying stress in diabetic human $\beta$ cells are planned.

Ellen P. Neff

Published online: 20 April 2020 https://doi.org/10.1038/s41684-020-0536-y 